\title{
Spezifische Immuntherapie rechnet sich
}

\section{Die Hyposensibilisierung ist die einzige kausale Allergietherapie. In einer gesundheitsökonomischen Studie wurde jetzt ihre Kosteneffek- tivität im Vergleich zur rein symptomatischen Behandlung überprüft.}

$E^{i}$ in Team aus Epidemiologen und Sozialmedizinern wollte die Kosteneffektivität der spezifischen subkutanen Immuntherapie (SCIT) im Rahmen der Gegebenheiten des deutschen Gesundheitswesens überprüfen. Dazu führte es eine gesundheitsökonomische Modellrechnung unter Einbeziehung von Literaturdaten zur Epidemiologie und $\mathrm{Pa}$ thophysiologie von Rhinitis und Asthma sowie zur Wirksamkeit der SCIT auf Basis von „Markov-Modellen“ durch. Den Berechnungen zugrundegelegt wurde der natürliche Verlauf der allergischen Erkrankung ohne Behandlung, also die Entwicklung von der milden Rhinitis zu schwereren Erkrankungsformen und die hohe Wahrscheinlichkeit, in späteren Jahren Asthma zu entwickeln. Die während dieses Verlaufs möglichen Ereignisse wie Verbesserung, Verschlechterung, Heilung oder Tod wurden mit einem Faktor für die Wahrscheinlichkeit des Eintretens versehen. Parallel wurden für die einzelnen Ereignisse durchschnittliche jährliche Kosten und Nutzen über 15 Jahre hinweg bestimmt. Die Kosten für die SCIT wurden auf Basis einer dreijährigen präsaisonalen Therapie festgelegt als $600 € \mathrm{im}$ ersten und je $540 €$ in den Folgejahren. Die Simulationsergebnisse pro Verlauf und Patient gingen schließlich in die inkrementelle Kosteneffektivität, das Verhältnis Kostendifferenz zu Wirksamkeitsdifferenz zweier Therapien, als Vergleich der Behandlung mit SCIT plus Symptomatika versus Therapie mit Symptomatika alleine ein.

Aus der gesellschaftlichen Perspektive war die Option SCIT plus Symptomatika mit einer jährlichen Kostenersparnis von 140 Euro pro Patient assoziiert. Nach zehnjähriger Krankheitsdauer lagen SCIT plus symptomatische Therapie, was die Kosten betrifft, gleich auf. rapie und die rein symptomatische The-
Eine Hyposensibilisierung spart Kosten und verbessert die Lebensqualität.

Die inkrementelle Kosteneffektivität betrug $-19.787 €$ pro "quality-adjusted life-year" (QALY), eine Kennzahl zur Bewertung eines Lebensjahres in Relation zur Gesundheit. Aus der Perspektive des Kostenträgers war die SCIT mit geringen Zusatzkosten verbunden. Die daraus resultierende inkrementelle Kosteneffektivität betrug im Durchschnitt $8.308 €$ pro QALY.

Fazit: Eine gesundheitsökonomische Berechnung aus einer gesamtgesellschaftlichen Perspektive ergab für eine zusätzlich zur symptomatischen Therapie durchgeführte SCIT ein verbessertes klinisches Ergebnis bei gleichzeitigen Kosteneinsparungen verglichen mit einer alleinigen symptomatischen Therapie. $b k$

Brüggenjürgen B et al. Cost-effectiveness of specific subcutaneous immunotherapy in patients with allergic rhinitis and allergic asthma. Ann Allergy Asthma Immunol 2008; 101: 316-24

\section{Rekombinantes Allergen bewährt sich}

\section{Rekombinante Allergene können in hoher Reinheit hergestellt wer- den und reduzieren so bei einer spezifischen Immuntherapie (SIT) das Risiko, durch Begleitsubstanzen zusätzliche Sensibilisierungen auszulösen. Wirksamkeit und Verträglichkeit einer SIT mit dem re- kombinanten Birkenpollenhauptallergen wurden jetzt untersucht.}

$\mathrm{n}$ einer multizentrischen randomisierten doppelblinden und plazebokontrollierten Studie wurden 134 Patienten mit einer Birkenpollenallergie entweder mit dem rekombinanten Birkenpollenallergen $\mathrm{rBet} \mathrm{v} 1 \mathrm{a}$, einem zugelassenen Birkenpollenextrakt, natürlichem Birkenpollenhauptallergen $\mathrm{nBet} v 1$ oder Plazebo behandelt. Die Allergene waren an Aluminiumhydroxid adsorbiert. In der zwölfwöchigen Aufdosierungsphase wurden die subkutanen Injektionen wöchentlich gegeben, dann erhielten die
Patienten einmal pro Monat eine Injektion mit jeweils einem Bet-v-1-Gehalt von $15 \mu \mathrm{g}$ in den Verumgruppen. Die Studie dauerte zwei Jahre.

Verglichen mit Plazebo besserten alle aktiven Behandlungen signifikant die Heuschupfensymptomatik ( $\mathrm{rBet} v \mathrm{v}$, $\mathrm{p}=0,0002 ;$ nBet $\mathrm{v} 1, \mathrm{p}=0,0006 ;$ Birkenextrakt, $p=0,0024)$, reduzierten den Bedarf an Symptomatika (rBet v 1, $\mathrm{p}=0,0011 ;$ nBet $\mathrm{v} 1, \mathrm{p}=0,0025$; Birkenextrakt, $p=0,0063)$ und verminderten die Hautreaktivität $(\mathrm{p}<0,0001)$. Par- allel zur klinischen Besserung stiegen die Bet-v-1-spezifischen IgG-Spiegel deutlich an, dabei war der Anstieg in der mit dem rekombinanten Allergen behandelten Gruppe am ausgeprägtesten. Bei drei der 29 mit dem natürlichen Extrakt behandelten Patienten waren neu aufgetretene spezifische IgE-Antikörper nachweisbar (spezifisches IgE gegen Bet v 2), dagegen bei keinem der mit rBet $\mathrm{v} 1$ oder mit nBet $v 1$ behandelten Patienten. Nebenwirkungen traten vergleichbar häufig in allen Behandlungsgruppen auf.

Fazit: Die Therapie mit rekombinantem Birkenpollenallergen $\mathrm{rBet} \mathrm{v} 1$ ist sicher, wirksam und induziert eine starke spezifische IgG-Immunantwort.

$b k$

Pauli $G$ et al. Efficacy of recombinant birch pollen vaccine for the treatment of birchallergic rhinoconjunctivitis. J Allergy Clin Immunol 2008; 122: 951-60 